\section{GREQAM}

Groupement de Recherche en Economie Quantitative d'Aix-Marseille - UMR-CNRS 6579

Ecole des Hautes études en Sciences Sociales Universités d'Aix-Marseille II et III
Document de Travail $n^{\circ} 2011-44$

Aggregate instability under balanced-budget

consumption taxes: a re-examination

Carine Nourry

Thomas Seegmuller

Alain Venditti

October 2011 


\title{
Aggregate instability under balanced-budget consumption taxes: a re-examination*
}

\author{
Carine NOURRY \\ Université de la Méditerranée and GREQAM \\ Thomas SEEGMULLER \\ CNRS - GREQAM \\ and \\ Alain VENDITTI \\ CNRS - GREQAM and EDHEC
}

\begin{abstract}
We re-examine the destabilizing role of balanced-budget fiscal policy rules based on consumption taxation. Using a one-sector model with infinitely-lived households, and assuming that preferences are of the Greenwood-Hercovitz-Huffman [8] (GHH) type, we show that non-linear consumption taxation may destabilize the economy, promoting expectation-driven fluctuations, if the tax rate is countercyclical. We also exhibit a Laffer curve, which explains the multiplicity of steady states when the tax rate is counter-cyclical. All these results are mainly driven by the absence of income effect. Finally, a numerical illustration shows that consumption taxation may be a source of instability for most OECD countries.
\end{abstract}

Keywords: Indeterminacy, endogenous business cycles, consumption taxes, balanced-budget rule, infinite-horizon model.

Journal of Economic Literature Classification Numbers: C62, E32, H20.

*This work was supported by French National Research Agency Grant (ANR-08-BLAN0245-01). We thank S. Bosi, R. Dos Santos Ferreira, J.P. Drugeon, F. Dufourt, J.M. Grandmont, J.T. Guo, C. Le Van, T. Lloyd-Braga, L. Modesto, Y. Vailakis and B. Wigniolle for useful comments and suggestions. This paper also benefited from presentations at the International Conference on "New issues on macroeconomic regulation: financial crisis, stabilisation policies and sustainable development", GREQAM, June, 2011, the "11th SAET Conference", Faro, Portugal, June 2011 and the "Workshop in honor of Cuong Le Van", Exeter, September 2011. 


\section{Introduction}

Balanced-budget rules recommendations to governments is a recurrent subject of controversies among economists and politicians. In the recent years, the financial crisis has generated a strong revival of interest of this debate through its consequences in terms of government debt sustainability. Some countries, characterized by too large debt and public deficits, are constrained to borrow on the financial markets against excessively high interest rates and this generates an increasing debt burden. In Europe, this has been experienced by several countries, starting with Greece, but US faces the same kind of difficulties. These concerns related to the solvability of public debt also enforce governments to reduce their deficits and implement public policies characterized by a balanced-budget.

From a theoretical point of view, several arguments have been considered in favor or against balanced-budget fiscal policy rules. In their seminal contribution, Schmitt-Grohé and Uribe [24] focus on the fact that balanced-budget rules may induce belief driven aggregate instability. Considering an economy with infinitely-lived households, their basic model introduces a proportional tax on labor income. Since public expenditures are assumed to be constant, the tax rate is counter-cyclical, which is shown to be a source of endogenous fluctuations. ${ }^{1}$

The economic intuition relies on self-fulfilling expectations. If households expect a higher tax rate at the next period, they will reduce their labor supply, which has a negative effect on capital returns. Therefore, they currently reduce investment. Needing less income to finance capital accumulation, they also supply less labor. Since the labor tax rate is counter-cyclical, it becomes larger, explaining that expectations are self-fulfilling.

Contributing to this debate, Giannitsarou [10] argues that consumption taxation should be preferred to income taxation. As in Schmitt-Grohé and Uribe [24], she assumes a balanced-budget rule with a constant level of public spendings, but entirely financed by consumption taxation, and she shows that saddle-path stability always prevails. ${ }^{2}$ Therefore, she suggests that a

\footnotetext{
${ }^{1}$ See also Gokan [7] and Pintus [21] for similar analysis of finance constrained economies.

${ }^{2}$ Consumption taxation is shown however to have destabilization effects by Lloyd-Braga
} 
government running policies with a stabilization target should increase the share of public spendings financed by consumption taxation.

Although Schmitt-Grohé and Uribe [24] and Giannitsarou [10] show that the (de-)stabilizing impacts of labor income and consumption taxations are opposite, both tax rates introduce a similar distortion, which affects the price of leisure in terms of the consumption good. An increase in either the labor income or the consumption tax rate decreases the relative price of leisure, reducing both labor supply and consumption through a substitution effect. As mentioned previously, this lowers labor supply and generates expectationdriven fluctuations and aggregate instability. In the case of consumption taxation, the decrease of consumption is also compensated by an increase of the labor supply through an income effect. When this latter effect is sufficiently large with respect to the former, any instability due to self-fulfilling expectations is ruled out. This explains the clear-cut conclusions obtained by Giannitsarou [10] in the case of additively-separable preferences. Nevertheless, this economic intuition also suggests that the final impact of a tax on consumption crucially depends on the relative size of the substitution and income effects, and thus on the specification of the utility function.

The aim of this paper is to re-examine the (de-)stabilizing role of consumption taxation considering the extreme case of the Greenwood-HercovitzHuffman [8] (GHH) utility function, characterized by the absence of any income effect. In addition, we assume a general one-sector technology to take into account any degree of input substitution. Finally, we focus on a balanced-budget rule that generalizes the one introduced in Giannitsarou [10]. We consider indeed public spendings that can be pro-cyclical, constant or counter-cyclical. Note that when public spendings are counter-cyclical, constant or weakly pro-cyclical, the consumption tax rate is counter-cyclical, whereas when public spendings are sufficiently pro-cyclical, the consumption tax rate becomes pro-cyclical.

Our main results are the following: First we prove the existence of a Laffer curve and thus of multiple steady states if the tax rate is counter-cyclical. Second, we show that the existence of local indeterminacy also requires a counter-cyclical consumption tax rate. More precisely, under GHH prefer-

et al. [17] in a finance constrained economy with heterogeneous households. 
ences, expectation-driven fluctuations arise for a non-empty range of tax rates when public spendings are constant, the case considered in Giannitsarou [10]. This conclusion is even reinforced by counter-cyclical public spendings: the minimum level of the tax rate compatible with local indeterminacy becomes lower.

A numerical illustration allows finally to confirm all these results. Using a standard parameterization of the fundamentals, we show that aggregate instability and endogenous sunspot fluctuations are compatible with tax rates observed in most OECD countries, depending on the degree of countercyclicality of the public spendings. This paper then proves that in the absence of any income effect, a balanced-budget rule financed by consumption taxation may be destabilizing. As a result, if the government's interventions have a stabilization target, any policy recommendation leading to an increase of the share of public spendings financed by consumption taxation may not be worthwhile. The precise impact of a larger tax rate on consumption has to be evaluated depending on the size of income effects.

The rest of this paper is organized as follows. We present the model in the next section. In Section 3, we analyze the multiplicity of steady states in connection with the existence of a Laffer curve. In Section 4, we give conditions for the existence of aggregate instability and endogenous sunspot fluctuations under GHH preferences. Section 5 focuses on a numerical illustration and in Section 6, we provide economic intuitions for our results. Some concluding remarks are provided in Section 7, and all the proofs are in a final Appendix.

\section{The model}

\subsection{The production structure}

Consider a perfectly competitive economy in which the final output is produced using capital $K$ and labor $L$. The production function of a representative firm is thus $A F(K, L)$, with $F(K, L)$ homogeneous of degree one and $A>0$ a scaling parameter. Denoting, for any $L \neq 0, x=K / L$ the capital stock per labor unit, we define the production function in intensive form as 
$A f(x)$.

Assumption 1. $f(x)$ is defined over $\mathbb{R}_{+}, \mathbf{C}^{r}$ over $\mathbb{R}_{++}$for $r$ large enough, increasing $\left(f^{\prime}(x)>0\right)$ and concave $\left(f^{\prime \prime}(x)<0\right)$. Moreover, there exist $M>0$ and $N>0$ such that $f^{\prime}(0)>M$ and $f^{\prime}(+\infty)<N$.

The rental rate of capital $r(t)$ and the wage rate $w(t)$ then satisfy:

$$
\begin{aligned}
r(t) & =A f^{\prime}(x(t)) \\
w(t) & =A\left[f(x(t))-x(t) f^{\prime}(x(t))\right]
\end{aligned}
$$

We can also compute the share of capital in total income:

$$
s(x)=\frac{x f^{\prime}(x)}{f(x)} \in(0,1)
$$

and the elasticity of capital-labor substitution:

$$
\sigma(x)=-\frac{(1-s(x)) f^{\prime}(x)}{x f^{\prime \prime}(x)}>0
$$

\subsection{Households' behavior}

We consider an economy populated by a large number of identical infinitelylived agents. We assume without loss of generality that the total population is constant and normalized to one. At each period a representative agent supplies elastically an amount of labor $l \in[0, \ell]$, with $\ell>1$ his endowment of labor. He then derives utility from consumption $c$ and leisure $\mathcal{L}=\ell-l$ according to a function $U(c, \mathcal{L} / B)$, where $B>0$ is a scaling parameter, ${ }^{3}$ which satisfies:

Assumption 2. $U(c, \mathcal{L} / B)$ is defined over $\mathbb{R}_{+} \times[0, \ell], \mathbf{C}^{r}$ over $\mathbb{R}_{++} \times(0, \ell)$ for $r$ large enough, increasing with respect to each argument and concave. Moreover, $\frac{U_{12}}{U_{1}} \frac{\mathcal{L}}{B}-\frac{U_{22}}{U_{2}} \frac{\mathcal{L}}{B} \neq 1, \lim _{X \rightarrow 0} X U_{2}(c, X) / U_{1}(c, X)=0$ and $\lim _{X \rightarrow+\infty} X U_{2}(c, X) / U_{1}(c, X)=+\infty$, or $\lim _{X \rightarrow 0} X U_{2}(c, X) / U_{1}(c, X)=+\infty$ and $\lim _{X \rightarrow+\infty} X U_{2}(c, X) / U_{1}(c, X)=0$.

\footnotetext{
${ }^{3}$ The constant $B$ is used to prove the existence of a normalized steady state which remains invariant with respect to preference parameters such that the elasticity of intertemporal substitution in consumption or the wage elasticity of the labor supply.
} 
We also introduce a standard normality assumption between consumption and leisure which ensures that the demands for these two goods are increasing functions of the agent's total income

Assumption 3. Consumption $c$ and leisure $\mathcal{L}$ are normal goods.

Actually, within these general properties for the utility function, we mainly consider in this paper the Greenwood-Hercovitz-Huffman [8] (GHH) formulation such that

$$
U(c, \mathcal{L} / B)=u(c+G(\mathcal{L} / B))
$$

with $u($.$) and G($.$) some increasing and concave functions. Such a speci-$ fication then satisfies Assumption 3 and implies that the marginal rate of substitution between consumption and leisure depends on the latter only as

$$
\frac{U_{2}(c, \mathcal{L} / B)}{B U_{1}(c, \mathcal{L} / B)}=G^{\prime}(\mathcal{L} / B) / B
$$

As this expression does not depend on consumption, there is no income effect associated with the agent's labor supply decision. Assumption 2 imposes the following properties:

Assumption 4. $\epsilon_{\mathcal{L} \mathcal{L}}^{G} \equiv-G^{\prime}(X) /\left(G^{\prime \prime}(X) X\right) \neq 1, \lim _{X \rightarrow 0} G^{\prime}(X) X=$ 0 and $\lim _{X \rightarrow+\infty} G^{\prime}(X) X=+\infty$, or $\lim _{X \rightarrow 0} G^{\prime}(X) X=+\infty$ and $\lim _{X \rightarrow+\infty} G^{\prime}(X) X=0 .^{4}$

Remark 1: Three other specifications of preferences are also quite popular in the literature:

- the additively separable formulation $U(c, \mathcal{L} / B)=u(c)+v(\mathcal{L} / B)$, with $u(c)$ and $v(\mathcal{L} / B)$ some positive increasing concave functions, ${ }^{5}$

- the King-Plosser-Rebelo [13] (KPR) formulation

$$
U(c, \mathcal{L} / B)=\frac{[c v(\mathcal{L} / B)]^{1-\theta}}{1-\theta}
$$

with $v(\mathcal{L} / B)$ a positive increasing function and $\theta \geq 0$,

\footnotetext{
${ }^{4}$ If $G(X)=X^{1-\gamma} /(1-\gamma)$ with $\gamma \geq 0$ the inverse of the elasticity of the function, the first part of Assumption 4 is satisfied when $\gamma \in[0,1)$ while the second part holds if $\gamma>1$.

${ }^{5}$ Giannitsarou [10] mainly uses such an additively separable specification with $u(c)=$ lnc, $v(\mathcal{L} / B)=-[\ell-\mathcal{L}) / B]^{1+\chi} /(1+\chi)$ and $\chi \geq 0$.
} 
- the linearly homogeneous formulation which is characterized by the share of consumption within total utility $\alpha(c, \mathcal{L} / B) \in(0,1)$ defined as follows:

$$
\alpha(c, \mathcal{L} / B)=\frac{U_{1}(c, \mathcal{L} / B) c}{U(c, \mathcal{L} / B)}
$$

As we are interested in finding conditions for local indeterminacy, i.e. the existence of a continuum of converging equilibrium paths, under reasonable (standard) parameterizations, we do not consider these formulations. Indeed, under Assumptions 1-3, it can be shown that for the additively separable and KPR specifications, local indeterminacy is always ruled out. This conclusion generalizes the main result of Giannitsarou [10] to any production function and any utility function, be it additively separable or KPR. Moreover, with the linear homogeneous specification, local indeterminacy is ruled out under constant government spendings, and requires extreme conditions with counter-cyclical government spendings. ${ }^{6}$

Since the population is constant and of unit size, we have $L=l$. The intertemporal maximization program of the representative agent is given as follows:

$$
\begin{array}{cl}
\max _{c(t), l(t), K(t)} & \int_{t=0}^{+\infty} e^{-\rho t} U(c(t),(\ell-l(t)) / B) \\
\text { s.t. } & h(c(t)) c(t)+\dot{K}(t)+\delta K(t)=r(t) K(t)+w(t) l(t) \\
& K(0)>0 \text { given }
\end{array}
$$

where $\rho>0$ is the discount factor, $\delta \in(0,1)$ the depreciation rate of capital and $K(0)$ the initial capital stock. In the budget constraint, $h(c)$ denotes one plus the tax rate on consumption. This function is assumed to have the following properties:

Assumption 5. $h(c):[0,+\infty) \rightarrow[1,+\infty)$ is continuous, and $C^{1}$ on $(0,+\infty)$.

Assumption 5 provides quite large degrees of freedom for the formulation of government income $\Omega(c)=h(c) c-c$. This allows us to consider in the same framework pro-cyclical $(\Omega(c) / c$ and $h(c)$ increasing), or counter-cyclical

\footnotetext{
${ }^{6}$ The proofs of these claims can be provided upon request.
} 
$(\Omega(c) / c$ and $h(c)$ decreasing) consumption tax rules. Let us introduce the following elasticity:

$$
\zeta(c)=\frac{h^{\prime}(c) c}{h(c)}
$$

The consumption tax rate is then pro-cyclical (counter-cyclical) if $\zeta$ is positive (negative).

We assume in the following that the representative agent considers as given the tax rate on consumption. Let us introduce the Hamiltonian in current value:

$$
\mathcal{H}=U(c(t),(\ell-l(t)) / B)+\lambda(t)[r(t) K(t)+w(t) l(t)-h(c(t)) c(t)-\delta K(t)]
$$

with $\lambda(t)$ the shadow price of capital $K(t)$. Considering the prices (1)-(2) and $h(c)$ as given, we derive the following first order conditions

$$
\begin{array}{r}
U_{1}(c(t),(\ell-l(t)) / B)=\lambda(t) h(c(t)) \\
(1 / B) U_{2}(c(t),(\ell-l(t)) / B)=\lambda(t) w(t) \\
\dot{\lambda}(t)=-\lambda(t)[r(t)-\rho-\delta]
\end{array}
$$

Any solution needs also to satisfy the transversality condition

$$
\lim _{t \rightarrow+\infty} e^{-\rho t} \frac{U_{1}(c(t), \ell-l(t))}{h(c(t))} K(t)=0
$$

\subsection{Government}

Taxes on consumption are used to finance an endogenous level of public spending $\mathcal{G}(t)$. Given the level of government income $\Omega(c(t))$ derived from the tax function, the government expenditures $\mathcal{G}(t)$ are endogenously determined by the following balanced-budget rule:

$$
\mathcal{G}(t)=\Omega(c(t))=h(c(t)) c(t)-c(t)
$$

We further assume that the public expenditures $\mathcal{G}(t)$ neither affect the consumers' preferences nor the production function.

In the following we will consider a sufficiently general formulation of public expenditures $\mathcal{G}(t)$ that encompasses the case of Giannitsarou [10], in which the government faces an exogenous stream of constant expenditure $\mathcal{G}(t)=\mathcal{G}$ that is financed by levying proportional taxes on consumption. Our formulation allows indeed for counter or pro-cyclical government spendings. We 
choose consumption $c$ as a proxy of the measure of the business cycle and we assume that public spending is a function of $c$, namely $\mathcal{G}(t) \equiv \mathcal{G}(c(t))$. Using our notations we get

$$
\mathcal{G}(c(t))=\Omega(c(t))=h(c(t)) c(t)-c(t)=\tau_{c}(c(t)) c(t)
$$

This expression endogenously determines the level of the consumption tax rate. Let us then denote:

$$
\eta \equiv \mathcal{G}^{\prime}(c) c / \mathcal{G}(c)
$$

We will say that public expenditures are counter(pro)-cyclical when $\eta<$ $0(\eta>0)$. The Giannitsarou's case of constant government spending is obtained when $\eta=0$. Using the balanced-budget rule it follows that:

$$
\zeta=\frac{\tau_{c}(\eta-1)}{1+\tau_{c}}
$$

The tax rate $\tau(c)=\mathcal{G}(c) / c$ is therefore counter-cyclical for $\eta<1$, pro-cyclical for $\eta>1$ and constant for $\eta=1$.

\subsection{Intertemporal equilibrium}

Under Assumptions 2 and 3, substituting equation (2) in (11) and solving (10)-(11) with respect to $c(t)$ and $l(t)$ gives consumption demand and labor supply functions $c(K(t), \lambda(t))$ and $l(K(t), \lambda(t))$. Using (1)-(2), we get equilibrium values for the rental rate of capital $r(t)$ and the wage rate $w(t)$ :

$$
\begin{aligned}
r(t) & =A f^{\prime}(x(t)) \equiv r(K(t), \lambda(t)) \\
w(t) & =A\left[f(x(t))-x(t) f^{\prime}(x(t))\right] \equiv w(K(t), \lambda(t))
\end{aligned}
$$

with $x(t)=K(t) / l(K(t), \lambda(t))$. From the budget constraint in the program (8) and (12), we finally derive the following system of differential equations in $K$ and $\lambda$ :

$$
\begin{aligned}
\dot{K}(t) & =r(K(t), \lambda(t)) K(t)+w(K(t), \lambda(t)) l(K(t), \lambda(t))-\delta K(t) \\
& -h(c(K(t), \lambda(t))) c(K(t), \lambda(t)) \\
\dot{\lambda}(t) & =-\lambda(t)[r(K(t), \lambda(t))-\rho-\delta]
\end{aligned}
$$

An intertemporal equilibrium is then a path $\{K(t), \lambda(t)\}_{t \geq 0}$, with $K(0)>0$, that satisfies equations (18) and the transversality condition (13). 


\section{$3 \quad$ Steady state analysis}

\subsection{Multiplicity of steady states and Laffer curve}

A steady state is a 4 -tuple $\left(K^{*}, l^{*}, x^{*}, c^{*}\right)$ such that $x^{*}=K^{*} / l^{*}$, which is solution of:

$$
\begin{aligned}
\delta+\rho & =A f^{\prime}(x) \\
h(c) c & =l[A f(x)-\delta x] \\
\frac{U_{2}(c,(\ell-l) / B)}{B U_{1}(c,(\ell-l) / B)} & =\frac{A\left[f(x)-x f^{\prime}(x)\right]}{h(c)}
\end{aligned}
$$

Obviously, when $M>\delta+\rho>N$ in Assumption 1, there is a unique $x=x^{*}$ satisfying (19). However, given $x=x^{*}$, nothing guarantees the existence nor the uniqueness of $c, l$ and $k$.

Lemma 1. Let Assumptions 1-3 and 5 hold with $M>\delta+\rho>N$ and $\zeta \neq-1$ for all $l \in(0, \ell)$. Then there exists at most one steady state if the consumption tax rate is pro-cyclical, i.e. $\zeta \geqslant 0$. Multiplicity requires $\zeta<0$, at least for some values of $l \in(0, \ell)$.

Proof: See Appendix 8.1.

In the case of the balanced-budget rule as given by (15) and the expression (16), we conclude that uniqueness is ensured as soon as $\eta \geq 1$ and multiplicity requires $\eta<1$.

The multiplicity of steady states can also be related to the existence of a Laffer curve. Using the balanced-budget rule as given by (15) and considering a given $x=x^{*}$, a steady state can be defined by a solution $\left(\tau_{c}, c, l\right)$ satisfying:

$$
\begin{aligned}
\mathcal{G}(c) & =\Omega(c)=\tau_{c} c \\
\left(1+\tau_{c}\right) c & =\left[A f\left(x^{*}\right)-\delta x^{*}\right] l \\
\left(1+\tau_{c}\right) \frac{U_{2}(c,(\ell-l) / B)}{B U_{1}(c,(\ell-l) / B)} & =A\left[f\left(x^{*}\right)-x^{*} f^{\prime}\left(x^{*}\right)\right]
\end{aligned}
$$

Substituting the labor supply $l$ derived from (23) into (24), we can define consumption as $c=c\left(\tau_{c}\right)$. Therefore, government spendings and incomes can be expressed as functions of $\tau_{c}$. We can then define the following elasticities 


$$
\epsilon_{\mathcal{G}}\left(\tau_{c}\right) \equiv \frac{d \mathcal{G}}{d \tau_{c}} \frac{\tau_{c}}{\mathcal{G}}, \quad \epsilon_{\Omega}\left(\tau_{c}\right) \equiv \frac{d \Omega}{d \tau_{c}} \frac{\tau_{c}}{\Omega}
$$

The existence of a Laffer curve is obtained if $\epsilon_{\Omega}\left(\tau_{c}\right)$ is a non-monotonic function of $\tau_{c}$ and the multiplicity of steady states can be analyzed by comparing $\epsilon_{\mathcal{G}}\left(\tau_{c}\right)$ and $\epsilon_{\Omega}\left(\tau_{c}\right)$ when $\tau_{c} \in[0,+\infty)$. With GHH preferences, from the elasticity of the $G($.$) function \epsilon_{\mathcal{L} L}^{G}$ defined in Assumption 4, we need to introduce $\epsilon_{l l}^{G}=-\epsilon_{\mathcal{L} \mathcal{L}}^{G} \mathcal{L} / l$ which also gives the elasticity of the labor supply with respect to the wage rate as $\epsilon_{\ell w}=-\epsilon_{l l}^{G}$. It follows that

$$
\epsilon_{\mathcal{G}}\left(\tau_{c}\right)=\frac{\eta \tau_{c}\left(\epsilon_{l l}^{G}-1\right)}{1+\tau_{c}}, \quad \epsilon_{\Omega}\left(\tau_{c}\right)=\frac{1+\tau_{c} G_{l l}^{G}}{1+\tau_{c}}
$$

We then derive the existence of a Laffer curve in the case of a constant elasticity of the labor supply: ${ }^{7}$

Proposition 1. Let $U(c, \mathcal{L} / B)=u(c+G(\mathcal{L} / B))$ with $G(\mathcal{L} / B)=-(\ell-$ $\mathcal{L})^{1+1 / \epsilon_{l l}^{G}} /\left(1+1 / \epsilon_{l l}^{G}\right)$, and Assumptions 1, 2, 4 hold with $M>\delta+\rho>N$. Then $\epsilon_{\Omega}\left(\tau_{c}\right) \gtreqless 0$ if and only if $\tau_{c} \lesseqgtr \tau_{c}^{*} \equiv-1 / \epsilon_{l l}^{G}$.

Proof: See Appendix 8.2.

The Laffer curve is illustrated by $\Omega\left(c\left(\tau_{c}\right)\right)$ on Figure 1. The number of steady states is determined by the intersections of the curves $\mathcal{G}\left(c\left(\tau_{c}\right)\right)$ and $\Omega\left(c\left(\tau_{c}\right)\right)$. As shown in Lemma 1 , when $\eta \geq 1$, i.e. $\zeta \geq 0$, we have $\epsilon_{\mathcal{G}}\left(\tau_{c}\right)<\epsilon_{\Omega}\left(\tau_{c}\right)$ for all $\tau_{c} \geq 0$ and there exists a unique steady state. On the contrary, if government spendings are too counter-cyclical with $\eta \ll 0$ and the initial value $\mathcal{G}(c(0))$ is too large, there is no steady state.

In the case of constant government spendings with $\mathcal{G}(t)=\mathcal{G}$, the existence of two steady states is generic as soon as $\mathcal{G}$ is not too large. Actually, Figure 1 shows that there exist $\eta<0$ and $\bar{\eta} \in(0,1)$ such that the same property holds when variable government spendings are considered with $\eta \in(\underline{\eta}, \bar{\eta})$ and $\epsilon_{l l}^{G}<-\eta /(1-\eta){ }^{8}$

\footnotetext{
${ }^{7}$ In the case of a general function $G(\mathcal{L} / B)$ the elasticity $\epsilon_{l l}^{G}$ depends on $\tau_{c}$ and the Laffer curve may have an unconventional shape with multiple local maxima.

${ }^{8}$ This inequality ensures $\epsilon_{\mathcal{G}}(+\infty)>\epsilon_{\Omega}(+\infty)$ and thus a second crossing of the curves $\mathcal{G}\left(c\left(\tau_{c}\right)\right)$ and $\Omega\left(c\left(\tau_{c}\right)\right)$. Note that the inequality is obviously satisfied if government spendings are counter-cyclical with $\eta \leq 0$.
} 


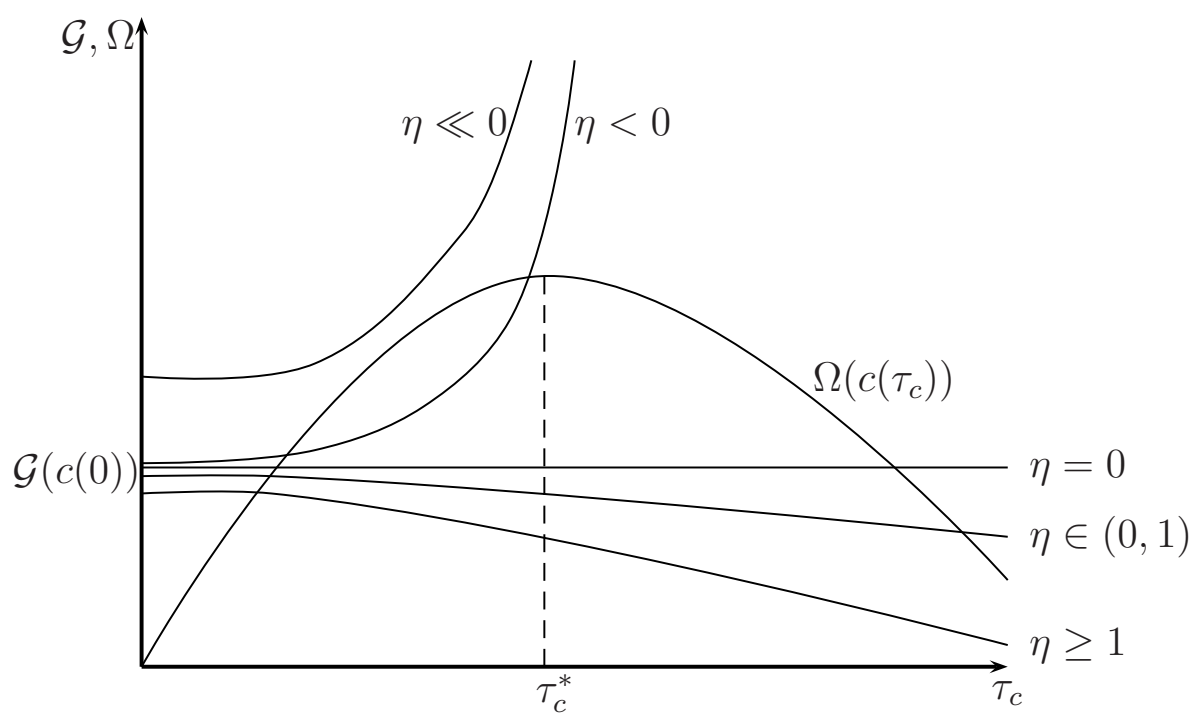

Figure 1: Laffer curve and multiple steady states

\subsection{A normalized steady state}

Consider equations (19)-(21). Assuming $\zeta\left(c^{*}\right) \neq-1$, equation (20) implicitely defines $c^{*}=c\left(x^{*}, l^{*}\right)$. Substituting this expression in (21), we use the scaling parameters $A>0$ and $B>0$ in order to give conditions for the existence of a normalized steady state (NSS in the sequel) such that $x^{*}=1$ and $l^{*}=1$. They have to satisfy:

$$
\begin{aligned}
\delta+\rho & =A f^{\prime}(1) \\
\frac{U_{2}(c(1,1),(\ell-1) / B)}{B U_{1}(c(1,1),(\ell-1) / B)} & =\frac{A\left[f(1)-f^{\prime}(1)\right]}{h(c(1,1))}
\end{aligned}
$$

Using this, we can show:

Proposition 2. Let Assumptions 1-3 and 5 hold and $\zeta\left(c^{*}\right) \neq-1$. Then there exist $A^{*}$ and $B^{*}$ which are the unique solutions of (27) and (28) respectively such that when $A=A^{*}$ and $B=B^{*},\left(x^{*}, l^{*}\right)=(1,1)$ is a steady state.

Proof: See Appendix 8.3. 
In the rest of the paper, we evaluate all the shares and elasticities previously defined at the NSS. From $(3),(4)$ and $c^{*}=c(1,1)$, we consider indeed $s(1)=$ $s, \sigma(1)=\sigma$ and $\zeta\left(c^{*}\right)=\zeta$.

Remark 2: Using a continuity argument we derive from Proposition 2 that there exists an intertemporal equilibrium for any initial capital stock $K_{0}$ in the neighborhood of $K^{*}$.

\section{Aggregate instability with consumption taxes}

Let us introduce the following elasticities:

$$
\epsilon_{c c}=-\frac{U_{1}(c, \mathcal{L})}{U_{11}(c, \mathcal{L}) c}, \quad \epsilon_{\mathcal{L} c}=-\frac{U_{2}(c, \mathcal{L})}{U_{21}(c, \mathcal{L}) c}, \quad \epsilon_{c \mathcal{L}}=-\frac{U_{1}(c, \mathcal{L})}{U_{12}(c, \mathcal{L}) \mathcal{L}}, \quad \epsilon_{\mathcal{L L}}=-\frac{U_{2}(c, \mathcal{L})}{U_{22}(c, \mathcal{L}) \mathcal{L}},
$$

As it is more convenient to study our model in terms of elasticities with respect to labor, we consider:

$$
\epsilon_{l c}=\epsilon_{\mathcal{L} c}, \epsilon_{c l}=-\epsilon_{c \mathcal{L}} \frac{\ell-l}{l}, \epsilon_{l l}=-\epsilon_{\mathcal{L L}} \frac{\ell-l}{l}<0
$$

The linearization of the dynamical system (18) around the NSS gives the characteristic polynomial $\mathcal{P}(\lambda)=\lambda^{2}-\lambda \mathcal{T}+\mathcal{D}$ with $\mathcal{T}$ and $\mathcal{D}$ which obviously depend on all the previous elasticities evaluated at the NSS. ${ }^{9}$

Our aim is to discuss the local indeterminacy properties of equilibria, i.e. the existence of a continuum of equilibrium paths starting from the same initial capital stock and converging to the NSS. Our model consists in one predetermined variable, the capital stock $K$, and one forward variable, the shadow price $\lambda$ of capital. Any solution from (18) that converges to the NSS satisfies the transversality condition and is an equilibrium. Therefore, given $K(0)$, if there is more than one initial price $\lambda(0)$ solution of equations (18), the equilibrium path from $K(0)$ will not be unique. In particular, if both roots of the characteristic polynomial have negative real parts, there will be a continuum of converging paths and thus a continuum of equilibria.

Definition 1. If $\mathcal{D}>0$ and $\mathcal{T}<0$ the NSS is locally indeterminate.

\footnotetext{
${ }^{9}$ The precise expression of the characteristic polynomial is given in Appendix 8.4.
} 
We provide immediately a general result.

Lemma 2. Under Assumptions 1-3 and 5, the NSS is locally indeterminate only if the consumption tax rate is counter-cyclical, i.e. $\zeta<0$.

We then derive from Lemmas 1 and 2 that if $\zeta \geq 0$, there exists a unique steady state which is locally determinate.

In the case of a $\mathrm{GHH}$ utility function, let us consider the elasticity of the function $G(\mathcal{L} / B)$, denoted $\epsilon_{\mathcal{L} \mathcal{L}}^{G}$, as defined in Assumption 4. We easily get from (29) that

$$
\epsilon_{c c}=\epsilon_{l c}, \frac{1}{\epsilon_{l l}}=\frac{1}{\epsilon_{c l}}+\frac{1}{\epsilon_{l l}^{G}}=-\frac{(1-s)(\delta+\rho)}{\delta(1-s)+\rho} \frac{1}{\epsilon_{c c}}+\frac{1}{\epsilon_{l l}^{G}}, \epsilon_{l l}^{G}=-\epsilon_{\mathcal{L} \mathcal{L}}^{G} \frac{\ell-\bar{l}}{\bar{l}}<0
$$

We derive the following results:

Proposition 3. Let $U(c, \mathcal{L})=u(c+G(\mathcal{L} / B)$ and Assumptions 1, 3 and 4 hold. When $\zeta=\tau_{c}(\eta-1) /\left(1+\tau_{c}\right)$ with $\eta<1$ and $\epsilon_{l l}^{G}<-\eta /(1-\eta)$, there exist $\bar{\sigma}>1, \bar{\rho}>0, \bar{\epsilon}_{c c}>0, \underline{\epsilon}_{c c}>0$ and $1>\bar{\tau}_{c}>\underline{\tau}_{c}>0$ such that the NSS is locally indeterminate if one of the following sets of conditions are satisfied:

i) if $\sigma \geq \bar{\sigma}, \eta<[\delta(1-s)+\rho] /(\rho \sigma), \epsilon_{c c} \geq \underline{\epsilon}_{c c}$ and $\tau_{c} \in\left(\underline{\tau}_{c}, \bar{\tau}_{c}\right)$,

ii) if $\sigma \in(0, \bar{\sigma}), \epsilon_{c c} \geq \max \left\{\underline{\epsilon}_{c c}, \bar{\epsilon}_{c c}\right\}$ and $\tau_{c} \in\left(\underline{\tau}_{c}, \bar{\tau}_{c}\right)$,

iii) if $\sigma \in(0, \bar{\sigma}), \rho \in(0, \bar{\rho}), \epsilon_{c c} \in\left(\underline{\epsilon}_{c c}, \bar{\epsilon}_{c c}\right)$ and $\tau_{c} \in\left(\underline{\tau}_{c}, \bar{\tau}_{c}\right)$.

Proof: See Appendix 8.5.

There is a range of tax rates such that indeterminacy occurs even when the balanced budget is characterized by constant public expenditures, i.e. $\eta=0$. This is in contrast to the result obtained in Giannitsarou [10] with additivelyseparable preferences. Note also that local indeterminacy is compatible with both pro-cyclical $(\eta \in(0,1))$ and counter-cyclical $(\eta<0)$ government spendings. It is worth pointing out that the upper bound $\bar{\tau}_{c}$ is lower than $\tau_{c}^{*}$ as given in Proposition 1, i.e. sunspot fluctuations arise in the increasing part of the Laffer curve, when government spendings are counter-cyclical $(\eta \leq 0)$.

\section{Constant/variable government spendings}

We provide now a numerical exercise to illustrate some of our results. On the basis of yearly data, we consider the benchmark parameterization $(s, \delta, \rho)=$ 
$(0.3,0.1,0.01)$. Concerning the other structural parameters, the numerical illustration will be based upon the following empirical evidences:

i) While Cobb-Douglas technologies are widely used in growth theory, recent papers have questioned the empirical relevance of this specification and find that capital and labor have an elasticity of substitution significantly different than unity. However, empirical evidences for both gross substitutability (elasticity above one) and gross complementarity (elasticity below one) of capital and labor are obtained in the literature. For instance, Duffy and Papageorgiou [4] report robust estimates that are contained in [1.24,3.24] and Krusell et al. [14] find an elasticity of substitution between unskilled labor and equipment of 1.67. On the contrary, Chirinko [3], Klump et al. [12] and León-Ledesma et al. [16] provide robust estimates in the range [0.4, 0.6]. In the following, in order to cover both configurations, we assume that $\sigma=2$ and $\sigma=0.6$.

ii) The elasticity of intertemporal substitution in consumption is usually assumed to be low, i.e. less than one. However, recent contributions provide divergent views. Mulligan [20], Vissing-Jorgensen [25] and Vissing-Jorgensen and Attanasio [26] repeatedly obtained estimates of this elasticity which are significantly larger than one. More recently, Gruber [10] and Kapoor and Ravi [11] provide robust estimates in the range $(2,3) .{ }^{10}$ We consider in the following $\epsilon_{c c}=2.2$ and $\epsilon_{c c}=2.8$.

iii) Concerning the elasticity of the labor supply with respect to the wage rate, Rogerson and Wallenius [23] have shown that the elasticities at the macroeconomic level are virtually unrelated to the micro elasticities, and are much larger than expected. The reason for this discrepancy is attributed to the role of the participation decision of women and the extent of early retirement. According to Rogerson and Wallenius [23] the macroeconomic elasticity of the labor supply with respect to the wage is in the range of $(2.25,3.0)$. We finally assume that $\epsilon_{\ell w}=-\epsilon_{l l}^{G}=2.5$.

Assuming first $\sigma=2$ and $\epsilon_{c c}=2.2$, Figure 5 shows the region of indeterminacy corresponding to Proposition 3, case iii), when $\tau_{c}$ is between $\underline{\tau}_{c}$ (dash line) and $\bar{\tau}_{c}$ (solid line).

\footnotetext{
${ }^{10}$ Barro [1] uses the lower bound of this interval to evaluate the welfare costs of rare disasters in a representative-consumer model.
} 


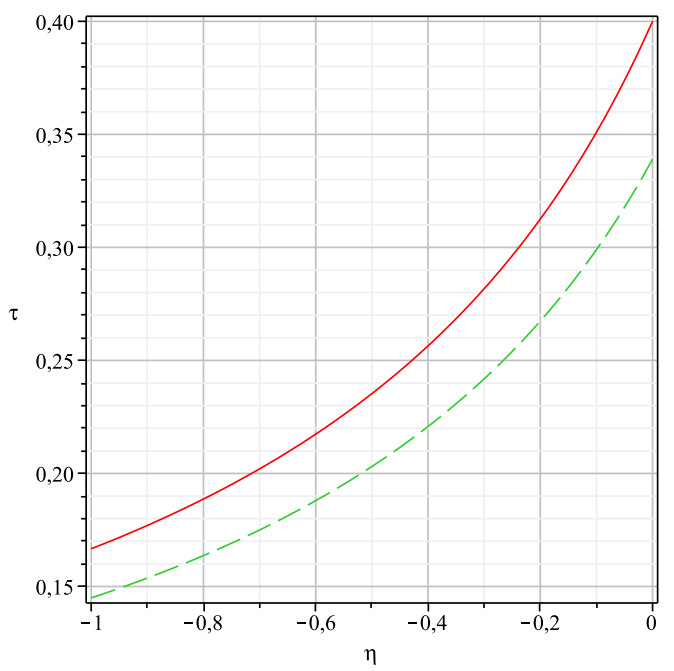

Figure 2: Indeterminacy, consumption taxes and public spendings with $\sigma>1$

We can see how the indeterminacy area is modified when the elasticity $\eta$ varies from -1 to 0 , thus from strongly counter-cyclical to constant public spendings. Considering consumption tax rates provided by Mendoza et al. $[18,19],{ }^{11}$ and more recently by Volkerink and De Haan [27], depending on the degree of counter-cyclicality of public spendings, local indeterminacy arises for almost all OECD countries. We get indeed:

\begin{tabular}{ll}
\hline \hline$\eta=0$ and $\tau \in(0.338,0.4)$ & Denmark, Norway \\
$\eta=-0.3$ and $\tau \in(0.241,0.282)$ & Ireland, Finland \\
$\eta=-0.36$ and $\tau \in(0.228,0.266)$ & Iceland, Luxembourg, Sweden \\
$\eta=-0.49$ and $\tau \in(0.204,0.237)$ & Austria, France \\
$\eta=-0.7$ and $\tau \in(0.175,0.202)$ & New Zealand, Portugal \\
$\eta=-0.8$ and $\tau \in(0.163,0.188)$ & Belgium, Germany, Greece, Netherlands, UK \\
$\eta=-1$ and $\tau \in(0.144,0.166)$ & Italy \\
\hline \hline
\end{tabular}

Note that Spain also belongs to the indeterminacy area when $\eta=-1.06$. On the contrary, Australia, Canada, Japan, Switzerland and the US are characterized by much lower consumption tax rates and are strongly out of the indeterminacy region, at least for reasonable values of the elasticity $\eta$.

\footnotetext{
${ }^{11}$ Updated estimates up to 1996 are available online from the authors.
} 
Assuming now $\sigma=0.6$ and $\epsilon_{c c}=2.8$, Figure 3 again shows the region of indeterminacy corresponding to Proposition 3, case $i i i$ ), when $\tau_{c}$ is between $\underline{\tau}_{c}$ (dash line) and $\bar{\tau}_{c}$ (solid line).

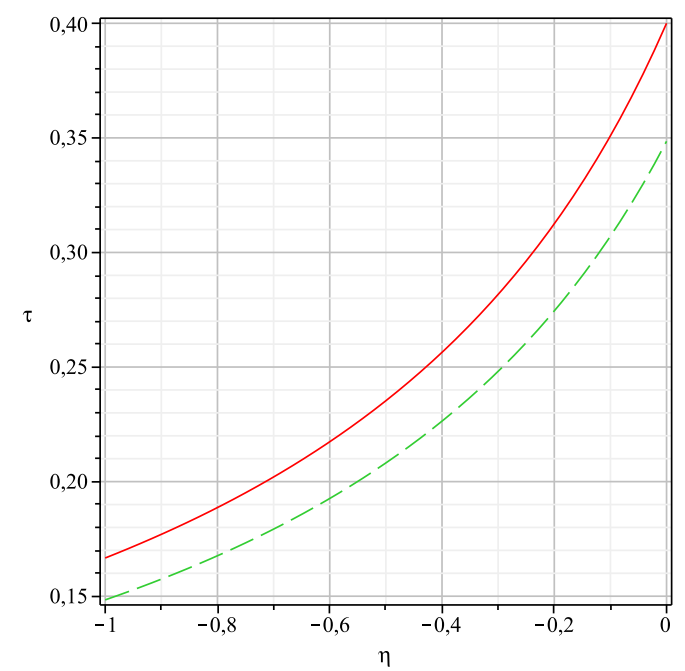

Figure 3: Indeterminacy, consumption taxes and public spendings with $\sigma<1$

Although we now consider a complementarity between capital and labor, local indeterminacy still arises for the same OECD countries as in the previous case. We get indeed:

\begin{tabular}{ll}
\hline \hline$\eta=0$ and $\tau \in(0.348,0.4)$ & Denmark, Norway \\
$\eta=-0.3$ and $\tau \in(0.248,0.282)$ & Ireland, Finland \\
$\eta=-0.36$ and $\tau \in(0.234,0.266)$ & Luxembourg, Sweden \\
$\eta=-0.49$ and $\tau \in(0.209,0.237)$ & Austria, France, Iceland \\
$\eta=-0.7$ and $\tau \in(0.179,0.202)$ & New Zealand, Portugal \\
$\eta=-0.8$ and $\tau \in(0.167,0.188)$ & Belgium, Germany, Greece, Netherlands, UK \\
$\eta=-1$ and $\tau \in(0.148,0.166)$ & Italy \\
\hline \hline
\end{tabular}

This numerical exercise clearly shows that the existence of indeterminacy is a robust property that occurs for a wide range of values for the elasticity of capital-labor substitution. This point is worth to mention as such a robustness is usually not satisfied. Indeed, in a Benhabib and Farmer [2] type model with productive externalities, Guo and Lansing [9] prove that local 
indeterminacy becomes much more difficult if not impossible when capital and labor are gross complement. ${ }^{12}$ Similarly, in a Schmitt-Grohé and Uribe [24] type model with labor taxes, Ghilardi and Rossi [5] show that balancedbudget rules deliver determinacy for a broad range of OECD countries when the elasticity of capital-labor substitution is in the range $[0.4,0.6]$.

Let us finally complete this exercise by discussing the value of $\eta$. Using annual data over the interval 1960 - 1998 for 22 OECD countries, Lane [15] provides some empirical estimates of the elasticity of government expenditure with respect to output growth. Since consumption is almost perfectly correlated with output, we then have a measure of $\eta$. Lane shows that in most OECD countries, namely Austria, Belgium, Finland, France, Germany, Italy, Netherlands, Sweden and UK, government spending is counter-cyclical, i.e. $\eta<0$. Moreover, his estimates perfectly match the values of $\eta$ used in the two previous tables for Finland, France, Sweden and UK. We have then proved that with GHH preferences, aggregate instability based of expectation-driven fluctuations can be generated by balanced-budget consumption taxes under empirically plausible parameters' configurations.

\section{Economic interpretations}

To establish the intuition for our results, let us explain why the expectations of an increase of the consumption tax rate may be self-fulfilling. To simplify, as in Schmitt-Grohé and Uribe [24] and Giannitsarou [10], we restrict our attention to a balanced-budget rule with constant spendings. In order to clearly emphasize the economic mechanism behind the occurrence of expectation-driven fluctuations, let us come back to the intratemporal choice between consumption and leisure. When both labor income and consumption are taxed, at rate $\tau_{l}$ and $\tau_{c}$ respectively, we have: ${ }^{13}$

$$
\frac{U_{2}(c, \ell-l)}{U_{1}(c, \ell-l)}=\frac{1-\tau_{l}}{1+\tau_{c}} w
$$

This equation shows that both tax rates affect the choice between consumption and leisure in a similar way. Indeed, an increase of $\tau_{l}$ or $\tau_{c}$ reduces

\footnotetext{
${ }^{12}$ See also Pintus [22].

${ }^{13}$ To simplify the presentation, we omit the scaling parameter $B$ in this section.
} 
the price of leisure in terms of the consumption good, which decreases the labor supply. This is the main channel that explains expectation-driven fluctuations in Schmitt-Grohé and Uribe [24] where only labor taxation is considered. To be more specific, assume that households expect a higher labor tax rate at the next period. They will reduce their future labor supply. Therefore, the return on capital will be lower, reducing current investment. To sustain a lower investment, households need to work less today. Since taxation is counter-cyclical, this implies a higher tax rate, explaining that expectations are self-fulfilling.

Since we argue that consumption taxation can a priori have the same effect on labor supply than labor taxation, let us focus on the case considered in this paper where the consumption tax is the only distortion. The (inverse) labor supply is given by:

$$
w=\left(1+\tau_{c}\right) \frac{U_{2}(c, \ell-l)}{U_{1}(c, \ell-l)}
$$

On the one hand, given $\tau_{c}$ and $c$, this equation describes a positive relationship between $w$ and $l$ (under Assumption 3), as drawn in Figure 4. On the other hand, an increase in either $\tau_{c}$ or $c$ shifts the labor supply to the left, reducing labor at equilibrium.

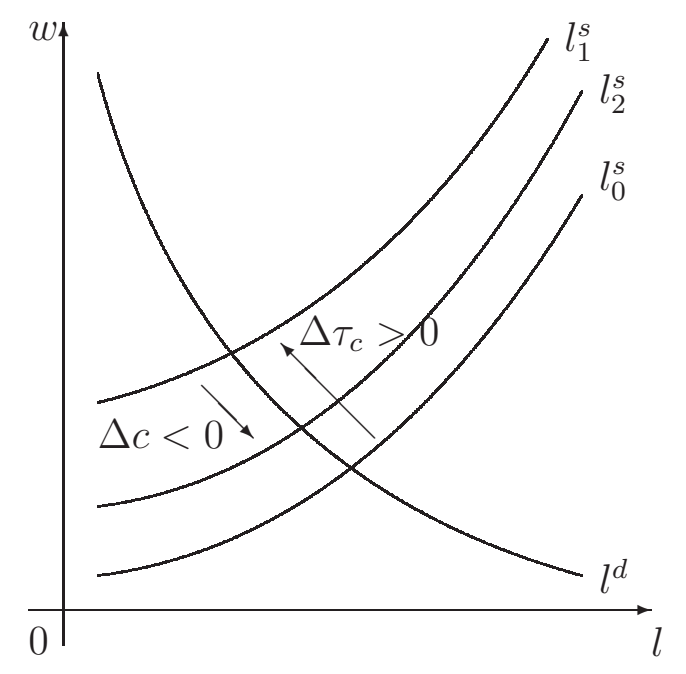

Figure 4: The role of labor market

If households expect a higher future consumption tax rate, they will reduce their future labor supply, as above. This corresponds to a substitution 
effect. The labor supply will move from $l_{0}^{s}$ to $l_{1}^{s}$ in Figure 4 . However, households also expect to decrease their future consumption. This induces an income effect, which goes in the opposite direction and increases labor at equilibrium. Indeed, as shown in Figure 4, this shifts the labor supply to the right from $l_{1}^{s}$ to $l_{2}^{s}$. However, if this second effect is dominated by the first one, a higher expected consumption tax rate implies a lower labor supply. This decrease of next period labor reduces the real interest rate, which has a negative effect on current investment. Needing less income today, households work less. This induces a lower consumption, and thus an increase of the current consumption tax rate in order to match the balanced-budget rule. Therefore, expectations are self-fulfilling.

These substitution and income effects, that play in opposite directions, allow to explain why indeterminacy may occur under some particular preferences, while this is not possible with others. Starting with the GHH case, equation (31) rewrites:

$$
w=\left(1+\tau_{c}\right) G^{\prime}(\ell-l)
$$

The absence of income effect in this formulation explains why indeterminacy appears easily. Consider as in Giannitsarou [10] the separable case, with a $\log$-linear utility of consumption, i.e. $U(c, \ell-l)=\ln c+v(\ell-l)$. Equation (31) becomes:

$$
w=\left(1+\tau_{c}\right) c v^{\prime}(\ell-l)=(c+\mathcal{G}) v^{\prime}(\ell-l)
$$

where the second equality is obtained using the balanced-budget rule. When $\mathcal{G}$ is constant, a modification of the tax rate does not have any impact on the labor supply. This explains that expectation-driven fluctuations cannot occur in this case. Actually, such a property still holds with general additivelyseparable preferences.

When the utility is of the King-Plosser-Rebelo [13] type, equation (31) is given by:

$$
w=\left(1+\tau_{c}\right) c \frac{v^{\prime}(\ell-l)}{v(\ell-l)}=(c+\mathcal{G}) \frac{v^{\prime}(\ell-l)}{v(\ell-l)}
$$

Again, the labor supply does not depend on the tax rate and expectationdriven fluctuations are also ruled out. Finally, when preferences are linear homogeneous, we can show that indeterminacy may occur, but this requires sufficiently counter-cyclical public spendings and a strong departure from the Cobb-Douglas specification. Indeed, for constant public expenditures and $U(c, \ell-l)=c^{\alpha}(\ell-l)^{1-\alpha}$, one obtains: 


$$
w=\left(1+\tau_{c}\right) c \frac{1-\alpha}{\alpha} \frac{1}{\ell-l}=(c+\mathcal{G}) \frac{1-\alpha}{\alpha} \frac{1}{\ell-l}
$$

and expectation-driven fluctuations are again ruled out.

\section{Concluding comments}

This paper re-examines the impact of balanced budget fiscal policy rules on the aggregate instability that may arise from self-fulfilling expectations. The recent debate has focussed indeed on two main contributions showing that while distortionary taxes on labor income could have expectation-driven destabilizing effects (Schmitt-Grohé and Uribe [24]), when the government finances instead its constant expenditures via taxes on consumption, the equilibrium is always unique and determinate (Giannitsarou [10]). Based on that, consumption taxes appear to be more appropriate than income taxes if the government is willing to avoid instability.

We show in this paper that such a conclusion has to be taken with caution as it heavily relies on the specification of the fundamentals, in particular the utility function. We prove indeed that GHH preferences, which are characterized by the absence of income effects, generate expectation-driven instability under counter-cyclical consumption taxes and plausible restrictions on the main parameters, namely, the elasticity of capital-labor substitution, the elasticity of intertemporal substitution in consumption and the elasticity of the labor supply. Moreover, numerical illustrations, based on empirically relevant parameterizations of the model, show that consumption taxation can be a source of instability for most OECD countries.

\section{Appendix}

\subsection{Proof of Lemma 1}

Let us consider equations (19)-(21). Equation (20) implicitly gives $c=$ $c\left(x^{*}, l\right)$, where

$$
\frac{d c}{d l} \frac{l}{c}=\frac{1}{1+\zeta}
$$

implying that $c$ can be defined as a function of $l$ for all $\zeta \neq-1$. Substituting $x=x^{*}$ and $c=c\left(x^{*}, l\right)$ into (21), one obtains: 


$$
H(l) \equiv h\left(c\left(x^{*}, l\right)\right) \frac{U_{2}\left(c\left(x^{*}, l\right),(\ell-l) / B\right)}{B U_{1}\left(c\left(x^{*}, l\right),(\ell-l) / B\right)}=A\left[f\left(x^{*}\right)-x^{*} f^{\prime}\left(x^{*}\right)\right]
$$

Multiplicity of equilibria may occur as soon as $H(l)$ is non-monotonic. Differentiating equation (33) with respect to $l$, we get:

$$
\epsilon_{H}(l) \equiv \frac{H^{\prime}(l) l}{H(l)}=\frac{1}{1+\zeta}\left[\frac{1}{\epsilon_{c c}}-\frac{1}{\epsilon_{l c}}+\frac{1}{\epsilon_{c l}}-\frac{1}{\epsilon_{l l}}+\zeta\left(1+\frac{1}{\epsilon_{c l}}-\frac{1}{\epsilon_{l l}}\right)\right]
$$

Under the normality Assumption 3 we conclude immediately that if $\zeta \geq 0$ $H(l)$ is monotonic and uniqueness holds.

\subsection{Proof of Proposition 1}

Let us consider equations (22)-(24). Equation (23) rewrites:

$$
l=\frac{\left(1+\tau_{c}\right) c}{A f\left(x^{*}\right)-\delta x^{*}} \equiv l\left(\tau_{c}, c\right)
$$

Substituting $l\left(\tau_{c}, c\right)$ into $(24)$, we get:

$$
\left(1+\tau_{c}\right) \frac{U_{2}\left(c,\left(\ell-l\left(\tau_{c}, c\right)\right) / B\right)}{B U_{1}\left(c,\left(\ell-l\left(\tau_{c}, c\right)\right) / B\right)}=A\left[f\left(x^{*}\right)-x^{*} f^{\prime}\left(x^{*}\right)\right]
$$

which defines $c$ as a function of $\tau_{c}$. Differentiating this equation gives:

$$
\frac{d c}{d \tau_{c}} \frac{\tau_{c}}{c}=-\frac{\tau_{c}}{1+\tau_{c}} \frac{1+\frac{1}{\epsilon_{c l}}-\frac{1}{\epsilon_{l l}}}{\frac{1}{\epsilon_{c c}}-\frac{1}{\epsilon_{l c}}+\frac{1}{\epsilon_{c l}}-\frac{1}{\epsilon_{l l}}}<0
$$

Using (22), we conclude that:

$$
\frac{d \Omega}{d \tau_{c}} \frac{\tau_{c}}{\Omega}=\frac{\frac{1}{\epsilon_{c c}}-\frac{1}{\epsilon_{l c}}+\frac{1}{\epsilon_{c l}}-\frac{1}{\epsilon_{l l}}-\frac{\tau_{c}}{1+\tau_{c}}\left(1+\frac{1}{\epsilon_{c l}}-\frac{1}{\epsilon_{l l}}\right)}{\frac{1}{\epsilon_{c c}}-\frac{1}{\epsilon_{l c}}+\frac{1}{\epsilon_{c l}}-\frac{1}{\epsilon_{l l}}} \equiv \epsilon_{\Omega}\left(\tau_{c}\right)
$$

Consider now the GHH utility function. We have $\epsilon_{c c}=\epsilon_{l c}$ and $1 / \epsilon_{c l}-1 / \epsilon_{l l}=$ $-1 / \epsilon_{l l}^{G}$. We conclude that:

$$
\epsilon_{\Omega}\left(\tau_{c}\right)=\frac{1+\tau_{c} \epsilon_{l l}^{G}}{1+\tau_{c}}
$$

with $\epsilon_{\Omega}(0)=1$ and $\epsilon_{\Omega}(+\infty)=\epsilon_{l l}^{G}<0$. Therefore, $\epsilon_{\Omega}\left(\tau_{c}\right) \lesseqgtr 0$ if and only if $\tau_{c} \gtreqless \tau_{c}^{*} \equiv-1 / \epsilon_{l l}^{G}$.

\subsection{Proof of Proposition 2}

To establish the existence of the normalized steady state $\left(x^{*}, l^{*}\right)=(1,1)$, we have to prove the existence and uniqueness of solutions $A^{*}$ and $B^{*}$ solving (27) and (28). We first obviously conclude that there is a unique value 
$A=(\delta+\rho) / f^{\prime}(1) \equiv A^{*}$ solving $(27)$. Considering $A^{*}$, we show now that there exists a unique $B^{*}$ solving

$$
\Gamma(B)=\frac{(\delta+\rho)[1-s(1)]}{h(c(1,1))}
$$

with

$$
\Gamma(B)=\frac{U_{2}(c(1,1),(\ell-1) / B)}{B U_{1}(c(1,1),(\ell-1) / B)}
$$

Under Assumption 2, either $\lim _{B \rightarrow 0} \Gamma(B)=0$ and $\lim _{B \rightarrow+\infty} \Gamma(B)=+\infty$ or $\lim _{B \rightarrow 0} \Gamma(B)=+\infty$ and $\lim _{B \rightarrow+\infty} \Gamma(B)=0$. Since Assumption 2 also ensures $\Gamma^{\prime}(B) B / \Gamma(B) \neq 0$, this proves existence and uniqueness of $B^{*}$.

\subsection{Proof of Lemma 2}

Considering all these elasticities evaluated at the NSS, the linearization of the dynamical system (18) around the NSS gives:

Proposition 8.1. Under Assumptions 1-2, the characteristic polynomial is

$$
\mathcal{P}(\lambda)=\lambda^{2}-\lambda \mathcal{T}+\mathcal{D}
$$

with

$$
\begin{aligned}
& \mathcal{D}=\frac{\delta+\rho}{\Delta} \frac{(1-s)[\delta(1-s)+\rho]}{s \sigma}\left[\frac{1}{\epsilon_{c c}}-\frac{1}{\epsilon_{l c}}-\frac{1}{\epsilon_{l l}}+\frac{1}{\epsilon_{c l}}-\zeta\left(\frac{1}{\epsilon_{l l}}-1-\frac{1}{\epsilon_{c l}}\right)\right] \\
& \mathcal{T}=\rho+\frac{(\delta+\rho)(1-s) \zeta}{\Delta \sigma \epsilon_{l c}}
\end{aligned}
$$

and

$$
\Delta=\left(\frac{1}{\epsilon_{c c}}+\zeta\right)\left(\frac{1}{\epsilon_{l l}}-\frac{s}{\sigma}\right)-\frac{1}{\epsilon_{c l} \epsilon_{l c}}
$$

Proof: Using (29) and the first order conditions (10) and (11), we get $\epsilon_{c l}=-\epsilon_{l c}(c h(c) / w l)$. Using the expression of $w$ at the NSS given in (17) together with (3) and (19) we find $w l=K(1-s)(\delta+\rho) / s$. Similarly we derive from $(20)$

$$
h(c) c+\delta K=(\delta+\rho) K / s \text { and thus } h(c) c=[\delta(1-s)+\rho] K / s
$$

Then at the NSS we get

$$
\epsilon_{c l}=-\frac{\delta(1-s)+\rho}{(1-s)(\delta+\rho)} \epsilon_{l c}
$$

Consider again the first order conditions (10) and (11). Under Assumptions 2 and 3, solving with respect to $c(t)$ and $l(t)$ gives consumption demand and labor supply functions $c(K(t), \lambda(t))$ and $l(K(t), \lambda(t))$. Using (29), the 
implicit function Theorem allows to get the partial derivatives of these functions evaluated at the NSS

$$
\begin{array}{ll}
\frac{d c}{d K}=\frac{c}{K \Delta} \frac{s}{\sigma \epsilon_{c l}}, & \frac{d c}{d \lambda}=-\frac{c}{\lambda \Delta}\left(\frac{1}{\epsilon_{l l}}-\frac{s}{\sigma}-\frac{1}{\epsilon_{c l}}\right) \\
\frac{d l}{d K}=-\frac{l}{K \Delta} \frac{s}{\sigma}\left(\frac{1}{\epsilon_{c c}}+\zeta\right), & \frac{d l}{d \lambda}=-\frac{l}{\lambda \Delta}\left(\frac{1}{\epsilon_{c c}}+\zeta-\frac{1}{\epsilon_{l c}}\right)
\end{array}
$$

with

$$
\Delta=\left(\frac{1}{\epsilon_{c c}}+\zeta\right)\left(\frac{1}{\epsilon_{l l}}-\frac{s}{\sigma}\right)-\frac{1}{\epsilon_{c l} \epsilon_{l c}}
$$

From these results and (17) we also derive at the NSS

$$
\begin{array}{ll}
\frac{d r}{d K}=-\frac{r(1-s)}{K \sigma}\left[1+\frac{s}{\Delta \sigma}\left(\frac{1}{\epsilon_{c c}}+\zeta\right)\right], & \frac{d r}{d \lambda}=-\frac{r(1-s)}{\lambda \Delta \sigma}\left(\frac{1}{\epsilon_{c c}}+\zeta-\frac{1}{\epsilon_{l c}}\right) \\
\frac{d w}{d K}=\frac{w s}{K \sigma}\left[1+\frac{s}{\Delta \sigma}\left(\frac{1}{\epsilon_{c c}}+\zeta\right)\right], & \frac{d w}{d \lambda}=\frac{w s}{\lambda \Delta \sigma}\left(\frac{1}{\epsilon_{c c}}+\zeta-\frac{1}{\epsilon_{l c}}\right)
\end{array}
$$

Consider then the system of differential equations in $K$ and $\lambda$ :

$$
\begin{aligned}
\dot{K}(t) & =r(K(t), \lambda(t)) K(t)+w(K(t), \lambda(t)) l(K(t), \lambda(t))-\delta K(t) \\
& -h(c(K(t), \lambda(t))) c(K(t), \lambda(t)) \\
\dot{\lambda}(t) & =-\lambda(t)[r(K(t), \lambda(t))-\rho-\delta]
\end{aligned}
$$

Linearization around the NSS using (40), (41) and the above results gives

$$
\begin{aligned}
& \frac{d \dot{K}}{d K}=\rho-\frac{(\delta+\rho)(1-s)}{\sigma}\left[\frac{1}{\epsilon_{c c}}+\zeta-\frac{1+\zeta}{\epsilon_{l c}}\right] \\
& \frac{d \dot{K}}{d \lambda}=-\frac{K(\delta+\rho)(1-s)}{\lambda \Delta s}\left[\frac{1}{\epsilon_{c c}}+\zeta-\frac{1}{\epsilon_{l c}}-(1+\zeta) \frac{\delta(1-s)+\rho}{(\delta+\rho)(1-s)}\left(\frac{1}{\epsilon_{l l}}-\frac{s}{\sigma}-\frac{1}{\epsilon_{c l}}\right)\right] \\
& \frac{d \dot{\lambda}}{d K}=\frac{\lambda(\delta+\rho)(1-s)}{K \sigma}\left[1+\frac{s}{\Delta \sigma}\left(\frac{1}{\epsilon_{c c}}+\zeta\right)\right] \\
& \frac{d \dot{\lambda}}{d \lambda}=\frac{(\delta+\rho)(1-s)}{\Delta \sigma}\left(\frac{1}{\epsilon_{c c}}+\zeta-\frac{1}{\epsilon_{l c}}\right)
\end{aligned}
$$

The expression of the characteristic polynomial follows after tedious computations and straightforward simplifications.

We may now prove Lemma 2. Note first that concavity of the utility function implies

while Assumption 3 implies

$$
\frac{1}{\epsilon_{c c} \epsilon_{l l}}-\frac{1}{\epsilon_{c l} \epsilon_{l c}} \leq 0
$$

$$
\frac{1}{\epsilon_{c c}}-\frac{1}{\epsilon_{l c}} \geq 0 \text { and } \frac{1}{\epsilon_{c l}}-\frac{1}{\epsilon_{l l}} \geq 0
$$

If $\zeta \geq 0$, we conclude that $\Delta<0$ and thus, from (42) and (43), that $\mathcal{D}<0$. 


\subsection{Proof of Proposition 3}

Using (30), we derive from Proposition 8.1

$$
\begin{aligned}
\mathcal{D} & =-\frac{\delta+\rho}{\Delta} \frac{(1-s)[\delta(1-s)+\rho]}{s \sigma}\left[\frac{1}{\epsilon_{l l}^{G}}+\zeta\left(\frac{1}{\epsilon_{l l}^{G}}-1\right)\right] \\
\mathcal{T} & =\rho+\frac{(\delta+\rho)(1-s) \zeta}{\Delta \sigma \epsilon_{c c}} \\
& =\frac{\rho \sigma\left(\frac{1}{\epsilon_{l l}^{G}}-\frac{s}{\sigma}\right)+\zeta\left[\rho \sigma \epsilon_{c c}\left(\frac{1}{\epsilon_{l l}^{G}}-\frac{s}{\sigma}\right)+\frac{(\rho+\delta)(1-s)}{\delta(1-s)+\rho}[\delta(1-s)+\rho(1-\sigma)]\right]}{\Delta \sigma \epsilon_{c c}}
\end{aligned}
$$

and

$$
\begin{aligned}
\Delta & =\frac{1}{\epsilon_{c c}}\left(\frac{1}{\epsilon_{l l}^{G}}-\frac{s}{\sigma}\right)+\zeta\left(\frac{1}{\epsilon_{l l}}-\frac{s}{\sigma}\right) \\
& =\frac{1}{\epsilon_{c c}}\left(\frac{1}{\epsilon_{l l}^{G}}-\frac{s}{\sigma}\right)+\zeta\left(\frac{1}{\epsilon_{l l}^{G}}-\frac{(1-s)(\delta+\rho)}{\delta(1-s)+\rho} \frac{1}{\epsilon_{c c}}-\frac{s}{\sigma}\right)
\end{aligned}
$$

Since $\zeta<0$, we derive that $\mathcal{T}<0$ requires $\Delta>0$, i.e.

$$
\zeta<-\frac{\frac{1}{\epsilon_{c c}}\left(\frac{1}{\epsilon_{l}^{G}}-\frac{s}{\sigma}\right)}{\frac{1}{\epsilon_{l l}^{G}}-\frac{s}{\sigma}-\frac{(1-s)(\delta+\rho)}{\delta(1-s)+\rho} \frac{1}{\epsilon_{c c}}} \equiv \bar{\zeta}
$$

Let us first consider the expression of $\mathcal{D}$ in (44). When $\Delta>0$, we get $\mathcal{D}>0$ if and only if

$$
\zeta>\frac{1}{\epsilon_{l l}^{G}-1} \equiv \underline{\zeta}_{1}
$$

We then need to show that $\underline{\zeta}_{1}<\bar{\zeta}$ which is satisfied if and only if

$$
\epsilon_{c c}>\frac{\left(1-\epsilon_{l l}^{G}\right)\left(\frac{s}{\sigma}-\frac{1}{\epsilon_{l}^{G}}\right)-\frac{(\rho+\delta)(1-s)}{\delta(1-s)+\rho}}{\left(\frac{s}{\sigma}-\frac{1}{\epsilon_{l l}^{G}}\right)} \equiv \underline{\epsilon}_{c c}>1
$$

Assume that $\zeta=\tau_{c}(\eta-1) /\left(1+\tau_{c}\right)$ with $\eta<1$. We derive that indeterminacy requires $\underline{\zeta}_{2}<\tau_{c}(\eta-1) /\left(1+\tau_{c}\right)<\bar{\zeta}$. This is obtained if $\epsilon_{l l}^{G}<-\eta /(1-\eta)$, $\epsilon_{c c}>\underline{\epsilon}_{c c}$ and $\tau_{c} \in\left(\underline{\tau}_{c}, \tau_{c}^{1}\right)$ with

$$
\tau_{c}^{1} \equiv-\frac{1}{(1-\eta) \epsilon_{l l}^{G}+\eta} \text { and } \underline{\tau}_{c} \equiv \frac{\left(\frac{s}{\sigma}-\frac{1}{\epsilon_{l l}^{G}}\right)}{\left[\epsilon_{c c}(1-\eta)-1\right]\left(\frac{s}{\sigma}-\frac{1}{\epsilon_{l l}^{G}}\right)+(1-\eta) \frac{(1-s)(\delta+\rho)}{\rho+(1-s) \delta}}
$$

Let us now consider the expression of $\mathcal{T}$ in (44). We get $\mathcal{T}<0$ if and only if

$\left(1+\tau_{c}\right)\left(\frac{1}{\epsilon_{l l}^{G}}-\frac{s}{\sigma}\right)<\tau_{c}(1-\eta)\left[\epsilon_{c c}\left(\frac{1}{\epsilon_{l l}^{G}}-\frac{s}{\sigma}\right)+\frac{(\rho+\delta)(1-s)}{\delta(1-s)+\rho} \frac{\delta(1-s)+\rho(1-\sigma)}{\rho \sigma}\right]$

a) Assume first that 


$$
\sigma \geq 1+\frac{\delta(1-s)}{\rho} \equiv \bar{\sigma}
$$

so that the rhs of (46) is negative. Moreover (46) can be written as follows

$$
\left(\frac{1}{\epsilon_{l l}^{G}}-\frac{s}{\sigma}\right)<\tau_{c}\left[\left[\epsilon_{c c}(1-\eta)-1\right]\left(\frac{1}{\epsilon_{l l}^{G}}-\frac{s}{\sigma}\right)+\frac{(\rho+\delta)(1-s)}{\delta(1-s)+\rho} \frac{\delta(1-s)+\rho(1-\sigma)}{\rho \sigma}\right]
$$

It follows that $\mathcal{T}<0$ if $\epsilon_{c c} \geq 1 /(1-\eta)$ and $\tau_{c}<\tau_{c}^{2}$ with

$$
\tau_{c}^{2} \equiv \frac{\left(\frac{s}{\sigma}-\frac{1}{\epsilon_{l}^{G}}\right)}{\left[\epsilon_{c c}(1-\eta)-1\right]\left(\frac{s}{\sigma}-\frac{1}{\epsilon_{l l}^{G}}\right)+\frac{(1-s)(\delta+\rho)}{\rho+(1-s) \delta}-\frac{(1-s)(\rho+\delta)}{\rho \sigma}}
$$

Note that $\tau_{c}^{2}>\underline{\tau}_{c}$ when $\eta<[\delta(1-s)+\rho] /(\rho \sigma)<1$, and that $\underline{\epsilon}_{c c}>1 /(1-\eta)$ when $\epsilon_{l l}^{G}<-\eta /(1-\eta)$.

b) Assume now that $\sigma \in(0, \bar{\sigma})$ and

$$
\epsilon_{c c}>\frac{\frac{(\rho+\delta)(1-s)+\rho}{\delta(1-\rho(1-s)+\rho(1-\sigma)]}}{\rho \sigma\left(\frac{s}{\sigma}-\frac{1}{\epsilon_{l l}^{G}}\right)} \equiv \bar{\epsilon}_{c c}
$$

so that the rhs of (46) is still negative. We derive from (47) that $\mathcal{T}<0$ in two distinct cases:

$$
\text { 1- if } \epsilon_{c c} \geq\left(1+\bar{\epsilon}_{c c}\right) /(1-\eta) \text { and } \tau_{c}<\tau_{c}^{2},
$$

2- if $\eta>-1 / \bar{\epsilon}_{c c}$ and $\epsilon_{c c} \in\left(\bar{\epsilon}_{c c}, 1+\bar{\epsilon}_{c c}\right)$ without any restriction on $\tau_{c}$.

c) Assume finally that $\sigma \in(0, \bar{\sigma})$ and $\epsilon_{c c} \leq \bar{\epsilon}_{c c}$. Then the rhs of (46) is positive and $\mathcal{T}<0$ without any restriction on $\tau_{c}$. But considering condition (45), we need to check that $\underline{\epsilon}_{c c}<\bar{\epsilon}_{c c}$, i.e.

$$
\rho s \epsilon_{l l}^{G 2}+\epsilon_{l l}^{G}[(\delta+\rho)(1-s)-\rho(\sigma+s)]-4 \rho^{2} \sigma s \equiv h(\rho)<0
$$

Direct inspection of this inequality shows that there exists $\bar{\rho} \in(0,+\infty]$ such that when $\rho \in(0, \bar{\rho}), \underline{\epsilon}_{c c}<\bar{\epsilon}_{c c}$.

Considering all the above computations, when $\epsilon_{l l}^{G}<-\eta /(1-\eta)$, local indeterminacy occurs in three different cases:

i) if $\sigma \geq \bar{\sigma}, \eta<[\delta(1-s)+\rho] /(\rho \sigma), \epsilon_{c c} \geq \underline{\epsilon}_{c c}$ and $\tau_{c} \in\left(\underline{\tau}_{c}, \bar{\tau}_{c}\right)$, with $\bar{\tau}_{c}=\min \left\{\tau_{c}^{1}, \tau_{c}^{2}\right\}$,

ii) if $\sigma \in(0, \bar{\sigma})$, and

- either $\epsilon_{c c} \geq \max \left\{\bar{\epsilon}_{c c},\left(1+\bar{\epsilon}_{c c}\right) /(1-\eta)\right\}$ and $\tau_{c} \in\left(\underline{\tau}_{c}, \bar{\tau}_{c}\right)$, with $\bar{\tau}_{c}=$ $\min \left\{\tau_{c}^{1}, \tau_{c}^{2}\right\}$,

- or $\eta>-1 / \bar{\epsilon}_{c c}, \epsilon_{c c} \in\left(\bar{\epsilon}_{c c},\left(1+\bar{\epsilon}_{c c}\right) /(1-\eta)\right)$ and $\tau_{c} \in\left(\underline{\tau}_{c}, \bar{\tau}_{c}\right)$, with $\bar{\tau}_{c}=\tau_{c}^{1}$,

iii) if $\sigma \in(0, \bar{\sigma}), \rho \in(0, \bar{\rho}), \epsilon_{c c} \in\left(\underline{\epsilon}_{c c}, \bar{\epsilon}_{c c}\right)$ and $\tau_{c} \in\left(\underline{\tau}_{c}, \bar{\tau}_{c}\right)$, with $\bar{\tau}_{c}=\tau_{c}^{1}$. The result is proved. 


\section{References}

[1] Barro, R. (2009): "Rare disasters, asset prices and welfare costs," American Economic Review 99, 243-264.

[2] Benhabib, J., and R. Farmer (1994): "Indeterminacy and increasing returns," Journal of Economic Theory 63, 19-41.

[3] Chirinko, R. (2008): "The CES production function in the theory and empirics of economic growth," Journal of Macroeconomics 30, 671-686.

[4] Duffy, J., and C. Papageorgiou (2000): "A cross-country empirical investigation of the aggregate production function specification," Journal of Economic Growth 5, 87-120.

[5] Ghilardi, M. and R. Rossi (2011): "Aggregate stability and balancedbudget rules," Working Paper, University of Surrey.

[6] Giannitsarou, C. (2007): "Balanced budget rules and aggregate instability: the role of consumption taxes," The Economic Journal 117, 14231435 .

[7] Gokan, Y. (2006): "Dynamic effects of government expenditure in a finance constrained economy," Journal of Economic Theory 127, 323333.

[8] Greenwood, J., Z. Hercovitz, and G. Huffman (1988): "Investment, capacity utilization and the real business cycle," American Economic Review 78, 402-417.

[9] Guo, J.T. and K. Lansing (2009): "Capital-labor substitution and equilibrium indeterminacy," Journal of Economic Dynamics and Control 33, 1991-2000.

[10] Gruber, J. (2006): "A Tax-based estimates of the elasticity of intertemporal substitution," NBER Working Paper 11945. 
[11] Kapour, M. and S. Ravi (2010): "Elasticity of intertemporal substitution in consumption: an empirical evidence from a natural experiment," Working Paper 21, 191-232.

[12] Klump, R., P. McAdam and A. Willman (2007): "Factor substitution and factor-augmenting technical progress in the United States: a normalized supply-side system approach," Review of Economics and Statistics 89, 183-192.

[13] King, R., C. Plosser, and S. Rebelo (1988): "Production, growth and business cycles," Journal of Monetary Economics 21, 191-232.

[14] Krusell, P., L. Ohanian, J.-V. Rios-Rull and G. Violante (2000): "Capital-skill complementarity and inequality: a macroeconomic analysis," Econometrica 68, 1029-1054.

[15] Lane, P. (2003): "The cyclical behaviour of fiscal policy: evidence from the OECD," Journal of Public Economics 87, 2661-2675

[16] León-Ledesma, M., P. McAdam and A. Willman (2010): "Identifying the elasticity of substitution with biased technical change," American Economic Review 100, 1330-1357.

[17] Lloyd-Braga, T., L. Modesto, and T. Seegmuller (2008): "Tax rate variability and public spending as sources of indeterminacy," Journal of Public Economic Theory 10, 399-421.

[18] Mendoza, E., G.M. Milesi-Ferretti and P. Asea (1997): "On the ineffectiveness of policy in altering long-run growth: Harberger's superneutrality conjecture," Journal of Public Economics 66, 99-126.

[19] Mendoza, E., A. Razin and L. Tesar (1994): "Effective tax rates in macroeconomics: Cross-country estimates of tax rates on factor incomes and consumption," Journal of Monetary Economics 34, 297-323.

[20] Mulligan, C. (2002): "Capital interest and aggregate intertemporal substitution," NBER Working Paper 9373. 
[21] Pintus, P. (2004): "Aggregate instability in the fixed-cost approach to public spending," University of Aix-Marseille, unpublished manuscript.

[22] Pintus, P. (2007): "Sunspots in real business-cycle models with complementary inputs," mimeo GREQAM.

[23] Rogerson, R. and J. Wallenius (2009): "Micro and macro elasticities in a life cycle model with taxes", Journal of Economic Theory 144, 22772292.

[24] Schmitt-Grohé, S. and M. Uribe (1997): "Balanced-budget rules, distortionary taxes, and aggregate instability", Journal of Political Economy 105, 976-1000.

[25] Vissing-Jorgensen, A. (2002): "Limited asset market participation and the elasticity of intertemporal substitution," Journal of Political Economy 110, 825-853.

[26] Vissing-Jorgensen, A. and O. Attanasio (2003): "Stock-market participation, intertemporal substitution and risk aversion," American Economic Review Papers and Proceedings 93, 383-391.

[27] Volkerink, B. and J. De Haan (2001): "Tax ratios: a critical survey," Empirica 29, 209-224. 